\title{
Quantification of occlusions influencing the tree stem curve retrieving from single-scan terrestrial laser scanning data
}

\author{
Peng Wan ${ }^{1,2} \mathbb{B}$, Tiejun Wang ${ }^{3}$, Wuming Zhang ${ }^{1 *}$, Xinlian Liang ${ }^{4}$, Andrew K. Skidmore ${ }^{3,5}$ and Guangjian Yan ${ }^{1}$
}

\begin{abstract}
Background: The stem curve of standing trees is an essential parameter for accurate estimation of stem volume. This study aims to directly quantify the occlusions within the single-scan terrestrial laser scanning (TLS) data, evaluate its correlation with the accuracy of the retrieved stem curves, and subsequently, to assess the capacity of single-scan TLS to estimate stem curves.

Methods: We proposed an index, occlusion rate, to quantify the occlusion level in TLS data. We then analyzed three influencing factors for the occlusion rate: the percentage of basal area near the scanning center, the scanning distance and the source of occlusions. Finally, we evaluated the effects of occlusions on stem curve estimates from single-scan TLS data.

Results: The results showed that the correlations between the occlusion rate and the stem curve estimation accuracies were strong $(r=0.60-0.83)$, so was the correlations between the occlusion rate and its influencing factors $(r=0.84-0.99)$. It also showed that the occlusions from tree stems were the main factor of the low detection rate of stems, while the non-stem components mainly influenced the completeness of the retrieved stem curves.

Conclusions: Our study demonstrates that the occlusions significantly affect the accuracy of stem curve retrieval from the single-scan TLS data in a typical-size $(32 \mathrm{~m} \times 32 \mathrm{~m})$ forest plot. However, the single-scan mode has the capacity to accurately estimate the stem curve in a small forest plot $(<10 \mathrm{~m} \times 10 \mathrm{~m})$ or a plot with a lower occlusion rate, such as less than $35 \%$ in our tested datasets. The findings from this study are useful for guiding the practice of retrieving forest parameters using single-scan TLS data.
\end{abstract}

Keywords: Stem curve, Stem volume, Terrestrial laser scanning, Scan mode

\section{Background}

The tree stem curve is defined as the relative rate of change in stem diameter with increasing tree height. It indicates the diameter at any height along the stem (Liang et al. 2013). Measurement of the stem curve is an important task in forestry, such as determining the inflexion points or cut points along the stem, calculating the total and merchantable stem volume, evaluating the quality of

\footnotetext{
* Correspondence: wumingz@bnu.edu.cn

${ }^{1}$ State Key Laboratory of Remote Sensing Science, Jointly Sponsored by Beijing Normal University and Institute of Remote Sensing and Digital Earth of Chinese Academy of Sciences; Beijing Engineering Research Centre for Global Land Remote Sensing Products, Institute of Remote Sensing Science and Engineering, Faculty of Geographical Science, Beijing Normal University, Beijing 100875, China

Full list of author information is available at the end of the article
}

stems and establishing the stem curve model (West 2009; Burkhart and Tomé 2012). In addition, the stem curve is an essential parameter used for accurate estimation of the above ground biomass of trees (Kankare et al. 2013; Yu et al. 2013; Stovall et al. 2017; Drew and Downes 2018). The stem curve of felled trees can be measured precisely using water displacement method or logging machines (Lundgren 2000; Özçelik et al. 2008). However, the stem curve of standing trees is hard to measure using traditional tools (Clark et al. 2000; West 2009).

Terrestrial laser scanning (TLS) is a promising technology for accurately retrieving stem curves because of its capability to document the 3D information of individual trees at the millimeter level (Dassot et al. 2011; Liang et al. 2019). Some studies have revealed high accuracies 
of the stem curve estimation using multi-scan TLS data (Liang et al. 2013), in which the reported root mean square error (RMSE) of the estimated stem curves were about $1.2 \mathrm{~cm}$. Pueschel et al. (2013) found that the stem attributes extracted from single-scan datasets had greater variability compared with those estimates from multi-scan datasets. However, deploying reflectors for the registration of multi-scan point clouds in natural forest with poor inter-visibility is labor-intensive and timeconsuming compared to the single-scan mode that point cloud registration is not required (Zhang et al. 2016a). In forest inventory tasks, time efficiency is a crucial issue especially when hundreds, even thousands of plots need to be measured routinely. Therefore, the use of the single-scan mode in forest inventory has received increasing attention (Liang et al. 2008, 2012; Lovell et al. 2011; Astrup et al. 2014; Kelbe et al. 2015; Olofsson and Olsson 2017). However, the single-scan mode has serious occlusion problems, which is an inherent limitation of the TLS applying in forest area (Watt and Donoghue 2005). The occlusion effect is prompted by the objects sheltering other objects of interest behind it in the direction of laser propagation (Abegg et al. 2017). The defective TLS data caused by the occlusion effect will lead to the inaccurate and unstable estimation of tree attributes (Van der Zande et al. 2006). Some studies have investigated how to reduce the occlusion effect by optimizing the arrangement of TLS positions (Trochta et al. 2013; Abegg et al. 2017; Wilkes et al. 2017). However, few studies have examined the occlusion effects in singlescan TLS data.

To systematically evaluate the influence of the stand condition, scan modes and point cloud processing approach on the extraction of tree attributes, Liang et al. (2018) launched an international benchmarking project on TLS methods for forestry applications. The main outcomes of the project indicated that the accuracy of estimated tree-level attributes from TLS data was mainly dependent on two factors: 1) the occlusion level of forest site; 2 ) the data processing method of tree attribute extraction. Here, the occlusion level is the possibility of trees being sheltered from the laser beam. The project's results showed that the accuracies of the stem curve retrieving from single-scan datasets were varied when using different data processing methods but greater variances were seen in different stand conditions (the stem density and the abundance of understory vegetation). This results indicated that the occlusion effect was more decisive than the performance of TLS data processing approaches for the estimation of tree attributes. In previous studies, the stand conditions were qualitatively categorized based on the stem density and the abundance of understory vegetation, which was not a quantitative and direct way. It is necessary to describe the stand conditions and occlusion effect quantitatively and analyze the impact of occlusions on the estimated stem curves.

This study aims to directly quantify the occlusions within the single-scan TLS data, evaluate its correlation with the accuracy of retrieved stem curves, and subsequently, to assess the capacity of single-scan TLS to estimate stem curves.

\section{Materials and methods TLS data}

The TLS data was provided by the Finish Geospatial Research Institute (FGI) for the international benchmarking of TLS methods for forestry application (Liang et al. 2018). The study area was located in southern Finland, and 24 plots were deployed with a fixed size of 32-by-32 m (Fig. 1). These plots varied in species, growth stages, and management activities and included both homogeneous and less-managed forests. The tree species included Scots pine (Pinus sylvestris), Norway spruce (Picea abies), silver (Betula pendula), and downy birches (Betula pubescens). The stand condition of these plots was described as "easy", "medium" and "difficult". The maximum stem density of the "easy", "medium" and "difficult" plots were 600, 1000 and 2000 stem.ha ${ }^{-1}$, respectively. In addition, the "easy" plot represents the plots with few understory vegetation; the "medium" plot represents the plots with sparse understory vegetation and the "difficult" plot represents the plots with dense understory vegetation.

The TLS data was collected in April/May 2014 using a Leica HDS6100 (Leica Geosystems AG, Heerbrugg, Switzerland) terrestrial laser scanner in multiscan mode. The multi-scan TLS datasets consist of five scans located at the center and in four quadrantal directions of each plot, and the single-scan datasets were the center scan of multi-scan datasets. For more details on the forest plots and data acquisition, please refer to Liang et al. (2018).

\section{Retrieving of stem curve from multi- and single-scan TLS datasets}

In this study, trees with a DBH larger than $5 \mathrm{~cm}$ were regarded as the targets of stem curve estimation. The stem curves of the target trees were measured both from single-scan datasets and multi-scan datasets. Datasets in both scan modes were processed with the same steps: 1) ground point filtering based on the cloth simulation filtering (CSF) method (Zhang et al. 2016b); 2) stem point extraction using an automatic method proposed by Zhang et al. (2019); additional manual editing was implemented to extract stem points accurately; 3) retrieving stem curves based on the extracted stem point using circle detection methods (Trochta et al. 2017). An 


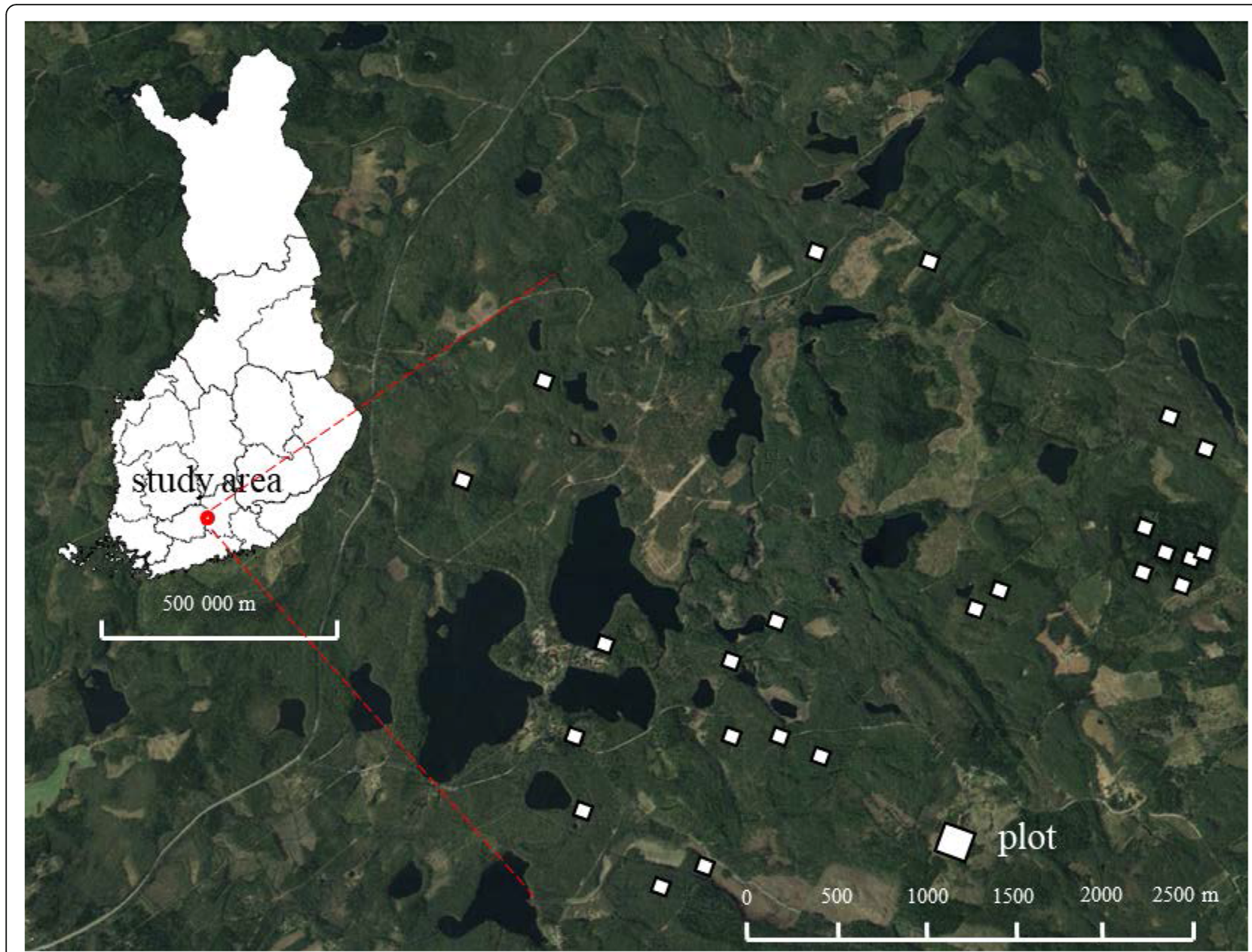

Fig. 1 The location of the study area and the sample plots in Finland (Liang et al. 2018)

overview of the workflow for retrieving the stem curve is shown in Fig. 2.

\section{Ground filtering}

To filter the ground points out from the TLS data, we adopted the CSF method implemented in CloudCompare (2.10. alpha, 2018) because it is fast, easy-to-use, and well performed. The CSF is a ground filtering algorithm based on the principle of cloth simulation (Zhang et al. 2016b). By iteratively dropping a simulated cloth on to an inverted (upside-down) LiDAR point cloud, the simulated cloth sticks to the ground points and bridges over the object points due to a certain degree of hardness of the simulated cloth. There are three main parameters need to be set for implementing the CSF algorithm, i.e., the cloth resolution, classification threshold and max iterations, which were set to $0.1 \mathrm{~m}, 0.1 \mathrm{~m}$ and 50 , respectively, in this study.

\section{Stem extraction}

To investigate the impact of occlusion effects on the stem curve estimation, the stem points need to be entirely and correctly extracted. Therefore, an automated algorithm (Zhang et al. 2019) plus manually editing was applied. The automated stem extraction algorithm is implemented using a program that developed on an open-source software CloudCompare (Girardeau-Montaut 2018). The automated algorithm identified the stem points by applying a segment-based classification strategy. First, the point cloud was thinned based on the different local curvature between stem points and points of other canopy elements. A part of branches and foliage points were removed by a threshold of local curvature. The local curvature was calculated by the formula of surface variation (Pauly et al. 2002) and the threshold was set to 0.1 in this study. Then, the remaining point cloud was segmented by connected component $(\mathrm{CC})$ labeling which is based on the proximity of points. In CC labeling, the point cloud was voxelized by 3D grids. The points in the adjacent grids that contained at least one point will be merged into the same segment. The vacant grids then became the gaps between the segments. After the CC labeling, the stem points were identified by the geometric feature of the segments, such as the 

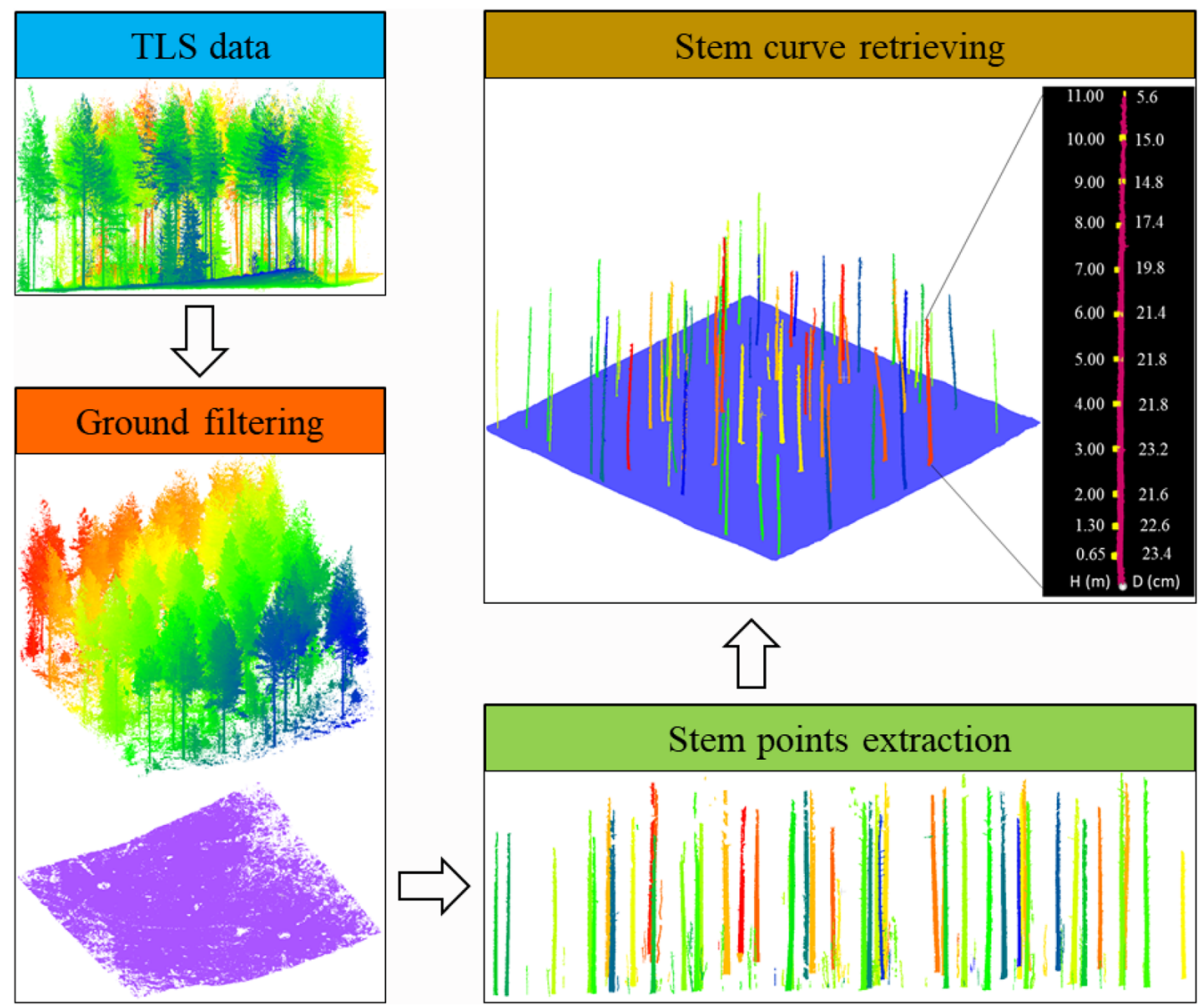

Fig. 2 An overview of the workflow for retrieving the stem curve

size and height-to-width ratio. The automated algorithm is accurate, fast and simple, however, some errors still have occurred. Therefore, the stem points were further manually refined through visual inspection.

\section{Stem curve retrieving}

We segmented the plot-level stem point cloud into individual stems and measured stem diameters at different heights above the ground, starting at $0.65 \mathrm{~m}$ and then by $1.3 \mathrm{~m}, 2 \mathrm{~m}$ and then for every next meter, until the maximum measurable height was reached (Liang et al. 2013; Trochta et al. 2017). We measured the diameters from $0.65 \mathrm{~m}$ because it is a general height of tree stump that left on the ground after tree felling by large sawing machines. It is also the start height for calculating the merchantable stem volume (Corral-Rivas et al. 2007; Kalantari et al. 2012). For measuring the stem diameters, circles were fitted in a $10-\mathrm{cm}$ horizontal slice that was projected on the horizontal plane at each corresponding height, and the diameters were measured through fitted circles. We used the Randomized Hough Transform (RHT) method (Xu et al. 1990; Xu and Oja 1993) to detect the circles on the stem in this study. The RHT method randomly selects three points from a point slice and calculates the circle parameter. This process is performed iteratively with a fixed number of iterations (200 in this study to balance the accuracy and speed). An accumulator is used to record these circle parameters. If the circle is similar to a circle in the accumulator, we replace the existing circle with the average of both circles and add 1 to its score. Otherwise, we insert the circle into an empty position in the accumulator and assign a score of 1 . Finally, the circle with the highest score is selected.

\section{Differences between the stem curve estimates from single- and multi-scan data}

To analyze the occlusion effect, stem curves from singlescan TLS data are compared to that from the multi-scan data. We assumed the estimates from the multi-scan data are complete and accurate enough to be used as a reference in this study because the stems were manually extracted. We used three types of indexes to assess the estimation accuracy in this study: 1) undetected rate, 2) completeness and 3) bias and RMSE. The undetected rate is the percentage of the reference stems that are not being detected in the single-scan TLS data. The completeness is the ratio of the number of measured diameters to the number of target height in percentage. The target height is dependent on the length of the stems extracted from the multi-scan TLS data. For the measured diameters on detected stems, the accuracy was assessed with regards to the bias, the relative bias and the RMSE, which were defined as 


$$
\begin{aligned}
& \text { Bias }=\frac{1}{n} \sum_{i=1}^{n}(y i-r y i) \\
& \text { Bias } \%=\frac{\text { Bias }}{\overline{r y}} \times 100 \% \\
& \text { RMSE }=\sqrt{\frac{\sum(y i-r y i)^{2}}{n}}
\end{aligned}
$$

where $y_{i}$ is the $i$ th measurement on a stem, $r y_{i}$ is the $i$ th reference diameter and $n$ is the number of measured diameters. Bias\% is the relative bias, $\overline{r y}$ is the mean of the reference diameters.

The undetected rate is a plot-level index. The completeness, bias and RMSE are tree-level indexes and their mean values of all trees in a plot are plot-level indexes.

\section{Occlusion evaluation}

\section{Calculating the occlusion rate}

The data deficient in single-scan TLS data is caused by the occlusions from different sources, e.g., tree stems, branches and foliage, and related to the stem density and scanning distance. Here, we proposed an index, occlusion rate, to quantify the overall occlusion degree of a single-scan TLS dataset. To calculate the occlusion rate, we projected the point cloud on the horizontal plane and rasterized the point cloud with $2 \mathrm{D}$ grids. Then the grids were binarized according to whether at least one point was included inside. The empty grids within the plot boundary were colored white, and the nonempty grids were colored black, as shown in Fig. 3. The percentage of the empty pixels within the plot was regarded as the occlusion rate. The grid size was set to $0.03 \mathrm{~m}$ in this study. The occlusion rate is not sensitive to the grid size because it is a percentage value of the number of grids. And according to our actual test, the change of grid size has less impact on the occlusion rate.

\section{Test plots selecting based on the occlusion rate}

We calculated the occlusion rates of all 24 single-scan plots. However, only a part of these plots was used in our tests because of the heavy workload involved in manual extraction of tree stems, even though the automatic method had been applied in advance. Test plots were selected based on the occlusion rates. A significant difference in occlusion rates among test plots was expected to make the trends in data emerged for exploring the influence of occlusion rates on the accuracy of stem curves. We reordered the plot IDs according to the occlusion rates. The plot with the lowest occlusion rate was first selected, and the following plots were selected when the cumulative change of the occlusion rate is larger than $2 \%$. Finally, 11 plots were chosen in this research. As shown in Fig. 4, the occlusion rates of the selected plots varied from $24.7 \%$ to $63.6 \%$, and the difference among them were significant. A visualization of the point cloud with different level of occlusion rates is shown in Fig. 5. The attributes of the selected plots are shown in Table 1.

\section{Stem density and its distribution}

The occlusion level in a dataset largely depends on the stem density and its distribution in the plot. In theory, the closer the stems with large DBH to the scan center, the higher the occlusion rate. Therefore, we used the percentage of basal area within $\mathrm{N}$ meters from the scanning center (PBA) to describe the stem density distribution near the scanning center. The $\mathrm{N}$ was set according to the plot size. In this study, the $\mathrm{N}$ was set as $5 \mathrm{~m}$ for the 32 -by-32 $\mathrm{m}$ plots. Since we have extracted the stems

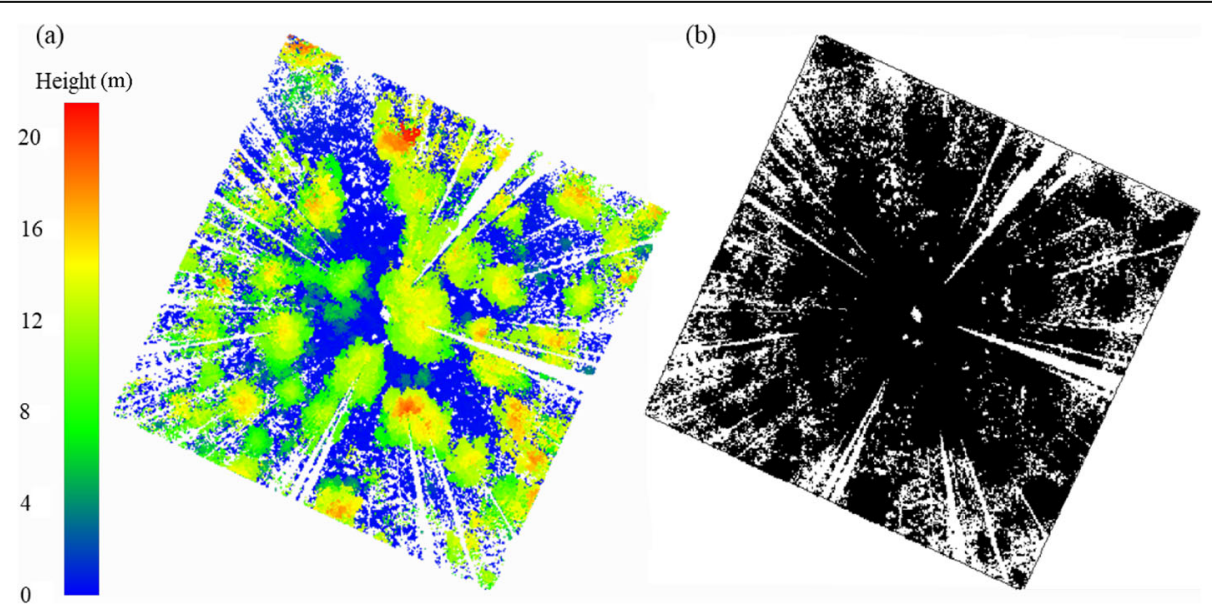

Fig. 3 Occlusion rate and its calculation. a The point cloud was converted to a 2D image from the top-view, the color from blue to red was filled according to the maximum height in the grids. $\mathbf{b}$ Image binarization. The white pixels within the plot denote empty grids. The percentage of the white pixels within the plot is the occlusion rate, which is $24.7 \%$ in this plot 


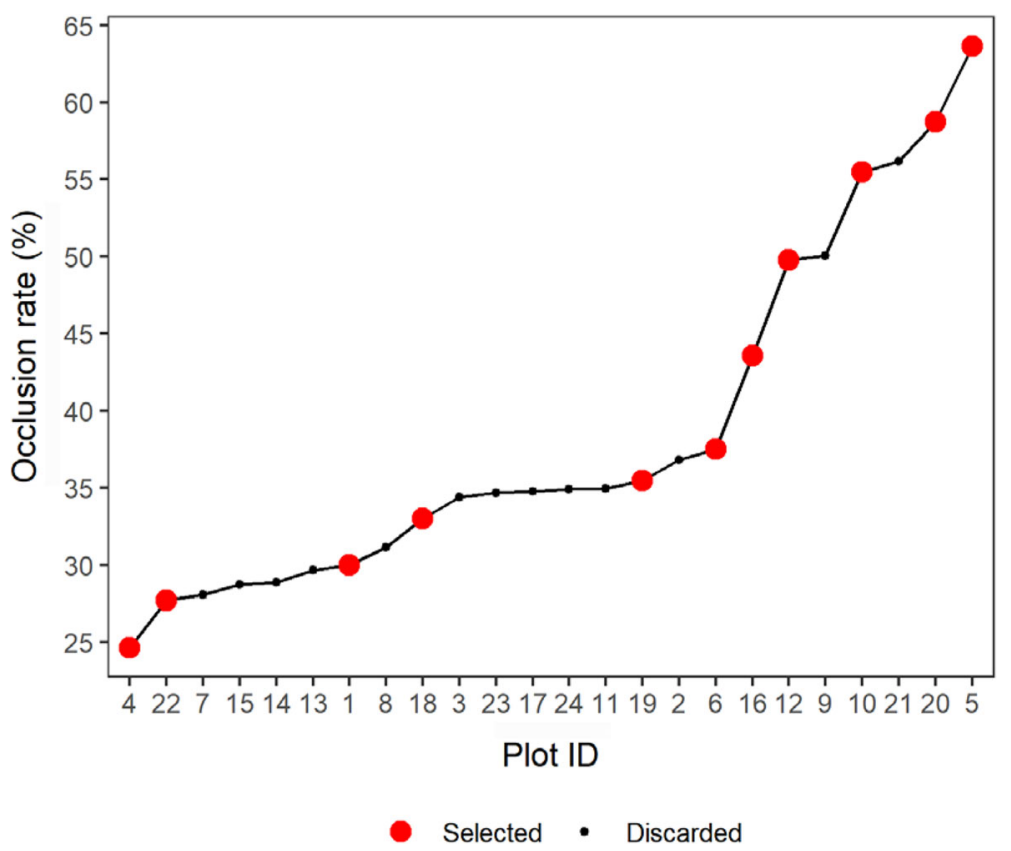

Fig. 4 Occlusion rates of all plots. The 11 red dots denote the plots selected for using in our experiments. The cumulative change between each two selected plots is large than $2 \%$

from the multi-scan datasets, the position and DBH of each stem were already available. Therefore, the total basal area in the plot and the basal area within $5 \mathrm{~m}$ from the scanning center can be directly calculated. The basal area and PBA were defined as

$$
\begin{aligned}
& \mathrm{BA} i=\frac{\pi \times(\mathrm{DBH} i / 2)^{2}}{10000} \\
& \mathrm{TBA}=\sum_{i=1}^{n} \mathrm{BA} i \\
& \mathrm{TBA}(\mathrm{N})=\sum_{i=1}^{m} \mathrm{BA} i
\end{aligned}
$$

$$
\mathrm{PBA}=\frac{\mathrm{TBA}(\mathrm{N})}{\mathrm{TBA}}
$$

where $i$ is the $i$ th stem in the plot, $n$ is the number of total stems in the plot, $m$ is the number of stems within $\mathrm{N}$ meters, TBA is the total BA of the plot and TBA(N) is the total BA within $\mathrm{N}$ meters.

\section{Identification of the occlusion sources}

The occlusion sources can be mainly categorized into two types in forests, i.e., the stems (S1) and non-stem components (S2). The S2 refers to the branches and foliage, the understory vegetation and artificial objects. Since we had a stem map with the locations and diameters of single trees in the plots (Fig. 6), the stems that

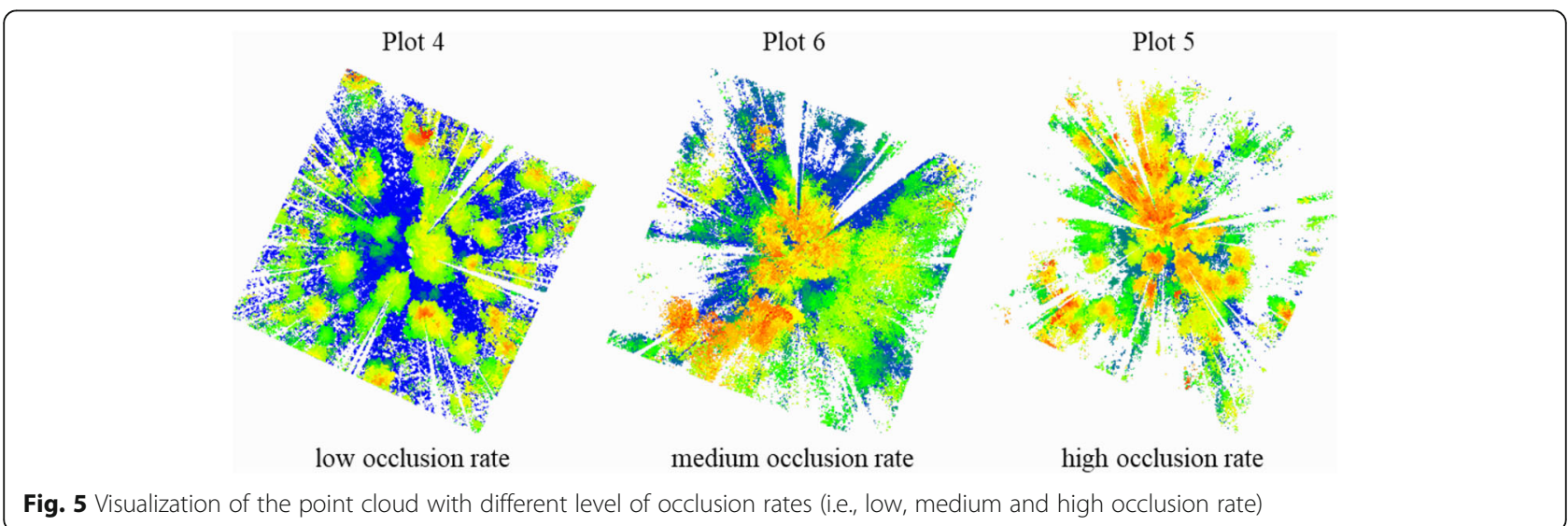


Table 1 The basic information on the selected plots in this study

\begin{tabular}{llll}
\hline Plot ID & Occlusion rate (\%) & Stem density $\left.\left(\text { stems }^{-1}\right)^{-1}\right)$ & Mean DBH(cm) \\
\hline 1 & 29.98 & 469 & 17.5 \\
4 & 24.66 & 762 & 12.7 \\
5 & 63.62 & 664 & 12.1 \\
6 & 37.54 & 557 & 18.0 \\
10 & 55.47 & 615 & 15.8 \\
12 & 49.76 & 1201 & 8.3 \\
16 & 43.56 & 527 & 22.9 \\
18 & 33.02 & 518 & 24.3 \\
19 & 35.46 & 293 & 22.8 \\
20 & 58.75 & 977 & 10.1 \\
22 & 27.7 & 566 & 14.5 \\
\hline
\end{tabular}

are totally or partly occluded by other stems can be labeled through laser beam tracing. Here, the totally occluded stems in the stem map were undetected caused by $\mathrm{S} 1$. However, there are other undetected stems not showed in the stem map are considered to be caused by $\mathrm{S} 2$. The diameters of the partly occluded stems caused by S1 may be severely underestimated because laser beams cannot penetrate the stems. Therefore, we used the absolute bias to describe the influence of the partly occlusions from S1. In contrast, the stems that seriously occluded by $\mathrm{S} 2$ would have very low completeness because of the dispersed distribution of branches and foliage. Therefore, the stems with low completeness $(<5 \%)$ are considered seriously occluded by non-stem components.

\section{Results}

\section{Accuracy of stem curve retrieving}

Accuracies of the retrieved stem curves were assessed using the three accuracy indices, which include the undetected rate, average completeness, and relative bias, as shown in Table 2. In addition, the number of undetected stems, mean bias and RMSE are also presented to show the estimation accuracy from another perspective. The undetected rates are at least $3.8 \%$, and up to $39.0 \%$. It shows that nearly half the plots lost more than 10 stems, all of which had a relatively high occlusion rate (Fig. 5). Plot 16 is a sole exception; it has a high occlusion rate, but only four stems were undetected. However, it has the highest RMSE among all the test plots. The average completeness range from $45.01 \%$ to $91.65 \%$. For the plots with large occlusion rates, their completeness of stem curve also appeared to be relatively low (less than $60 \%)$. The same trend is also found in relative bias, which ranges from $4.54 \%$ to $46.93 \%$. The mean biases of all the test plots are negative values, which indicate a general underestimation of the stem curve in single-scan datasets; and the underestimation ranged from 1 centimeter to over $8 \mathrm{~cm}$.

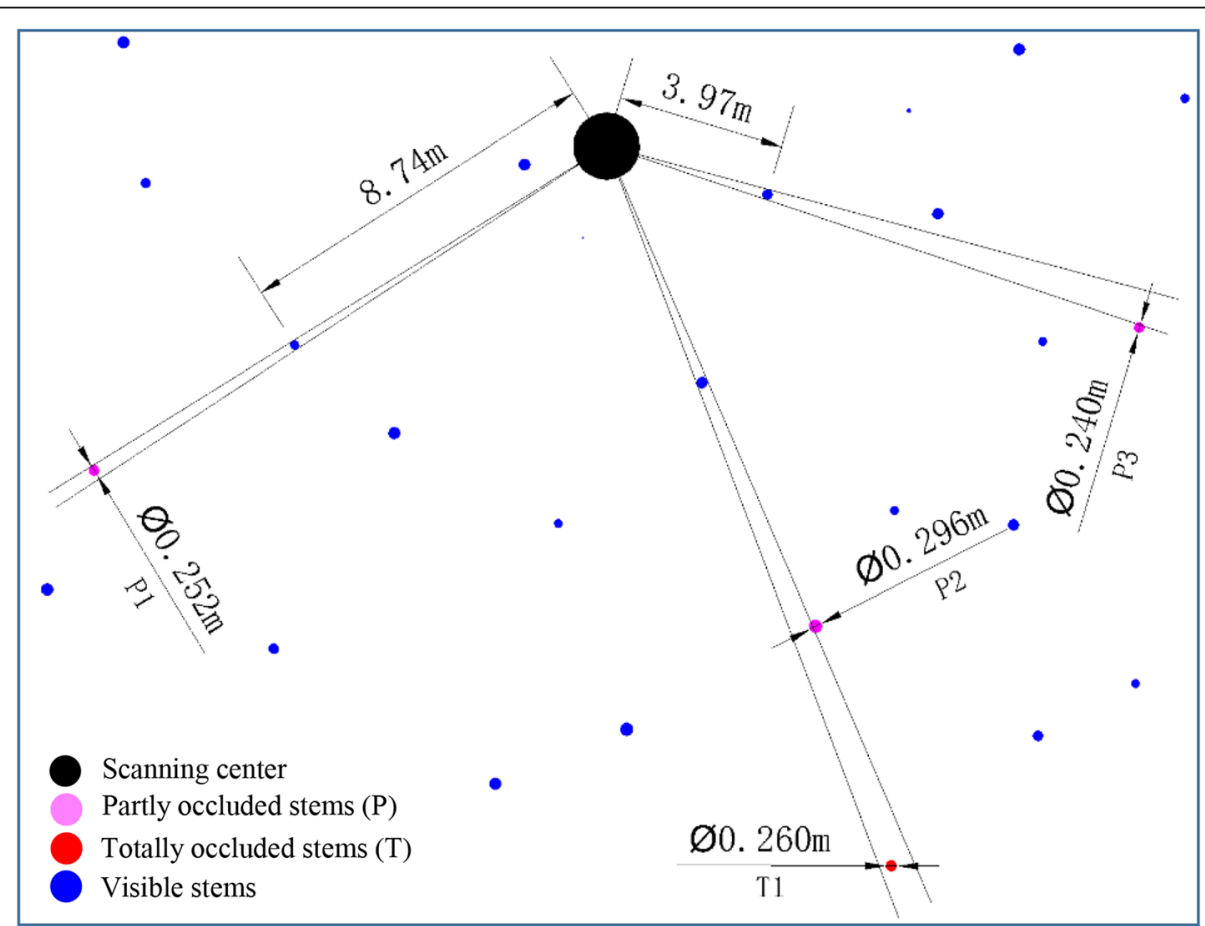

Fig. 6 A part of a stem map and the occlusions caused by stems. One stem was totally occluded ( $\mathrm{T} 1)$, and three stems were partly occluded ( $\mathrm{P}$, P2, P3). D denotes the DBH of the stem. The two distances are labeled to show the scale of this map 
Table 2 The retrieving accuracy of stem curve from single-scan TLS datasets

\begin{tabular}{|c|c|c|c|c|c|c|}
\hline Plot ID & Undetected rate (\%) & Average completeness (\%) & Relative bias (\%) & Number of undetected stem & Mean bias $(\mathrm{cm})$ & RMSE $(\mathrm{cm})$ \\
\hline 1 & 6.3 & 65.48 & 22.48 & 3 & -3.938 & 4.198 \\
\hline 4 & 7.7 & 73.74 & 19.76 & 6 & -2.508 & 3.256 \\
\hline 5 & 38.2 & 47.87 & 33.58 & 26 & -4.077 & 3.192 \\
\hline 6 & 26.3 & 47.09 & 46.93 & 15 & -8.464 & 5.514 \\
\hline 10 & 20.6 & 45.01 & 42.85 & 13 & -6.780 & 4.298 \\
\hline 12 & 16.3 & 51.81 & 38.71 & 20 & -3.206 & 1.917 \\
\hline 16 & 7.4 & 75.82 & 19.19 & 4 & -4.388 & 5.980 \\
\hline 18 & 3.8 & 78.45 & 17.75 & 2 & -4.309 & 4.313 \\
\hline 19 & 10.0 & 91.65 & 4.54 & 3 & -1.034 & 4.061 \\
\hline 20 & 39.0 & 55.45 & 37.21 & 39 & -3.756 & 2.502 \\
\hline 22 & 5.2 & 85.81 & 16.46 & 3 & -2.394 & 2.489 \\
\hline Mean & 16.4 & 65.29 & 27.22 & 13 & -4.078 & 3.793 \\
\hline
\end{tabular}

\section{Correlation between the occlusion rate and the estimation accuracy}

To further quantify the relations between occlusion rate and the accuracy indices, the Pearson correlation coefficient and the $p$-value were used. The occlusion rate is significantly correlated with the accuracy indices. The correlation coefficient between the undetected rate and the occlusion rate reached 0.83 , and the $p$-value is 0.001 (Fig. 7). As expected, the completeness of the stem curve is negatively correlated with the occlusion rate, and the correlation coefficient is -0.70 , which is relatively lower than that for the undetected rate. The relative bias has the lowest correlation degree $(r=0.60, p=0.048)$ with the occlusion rate among the accuracy indices, however, there is still an observed correlation between them.

\section{Influence of the stem density and its distribution}

A correlation matrix was generated to evaluate the correlations between the occlusion factors and the accuracy indices and the occlusion rate. As shown in Fig. 8, the stem density and PBA, influenced the accuracy assessment indexes to different degrees. The PBA is highly related to the accuracy indices, especially for the undetected rate $(r=0.92)$ which is followed by the correlation to the completeness $(r=-0.73)$ and relative bias $(r=0.67)$. The correlation between the stem density and the accuracy indices is weaker than the PBA correlation with the accuracy indices; the correlation coefficients range from 0.41 to 0.56 . Furthermore, PBA is also the most influential factor on the occlusion rate $(r=0.84)$, compared to the stem density $(r=0.44)$. In addition, strong correlations are also showed among accuracy indices; the relative bias is highly correlated to the average completeness $(r=-0.96)$. The correlations between the undetected rate and the relative bias as well as the average completeness are 0.69 and -0.74 , respectively.

\section{Influence of the scanning distance}

We collected the scanning distance of the individual trees (589 in total) in all test plots, and the undetected
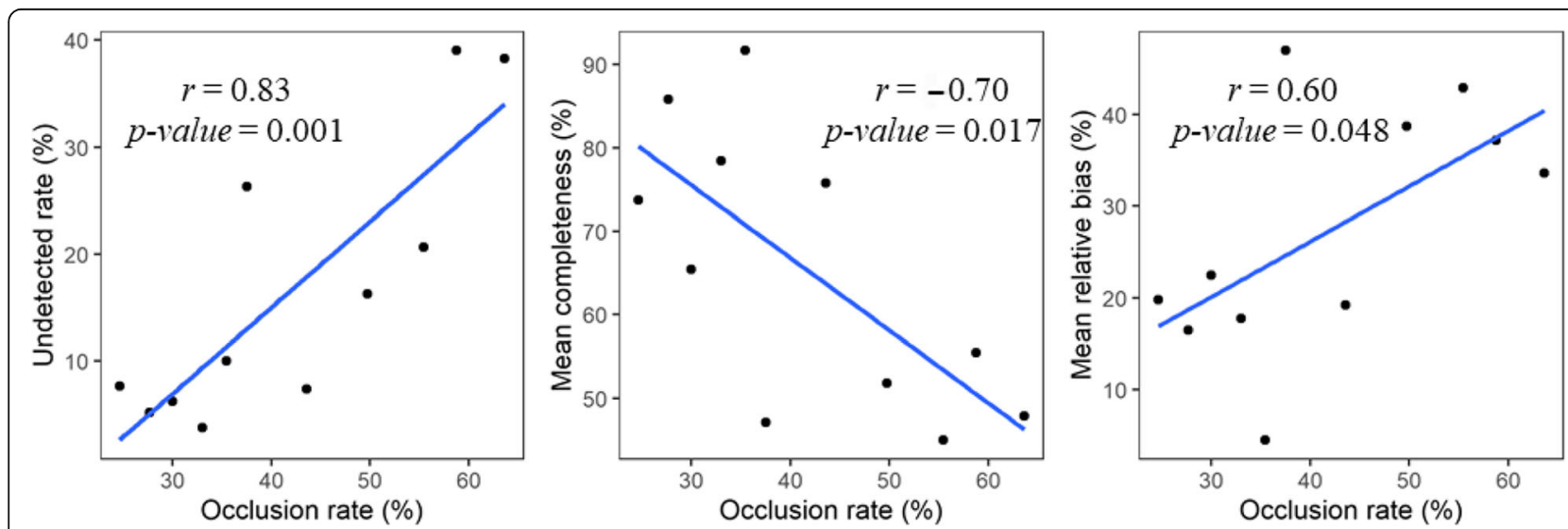

Fig. 7 Correlations between the occlusion rate and the three accuracy indices (i.e., undetected rate, mean completeness, and mean relative bias), $r$ denotes the Pearson correlation coefficient 


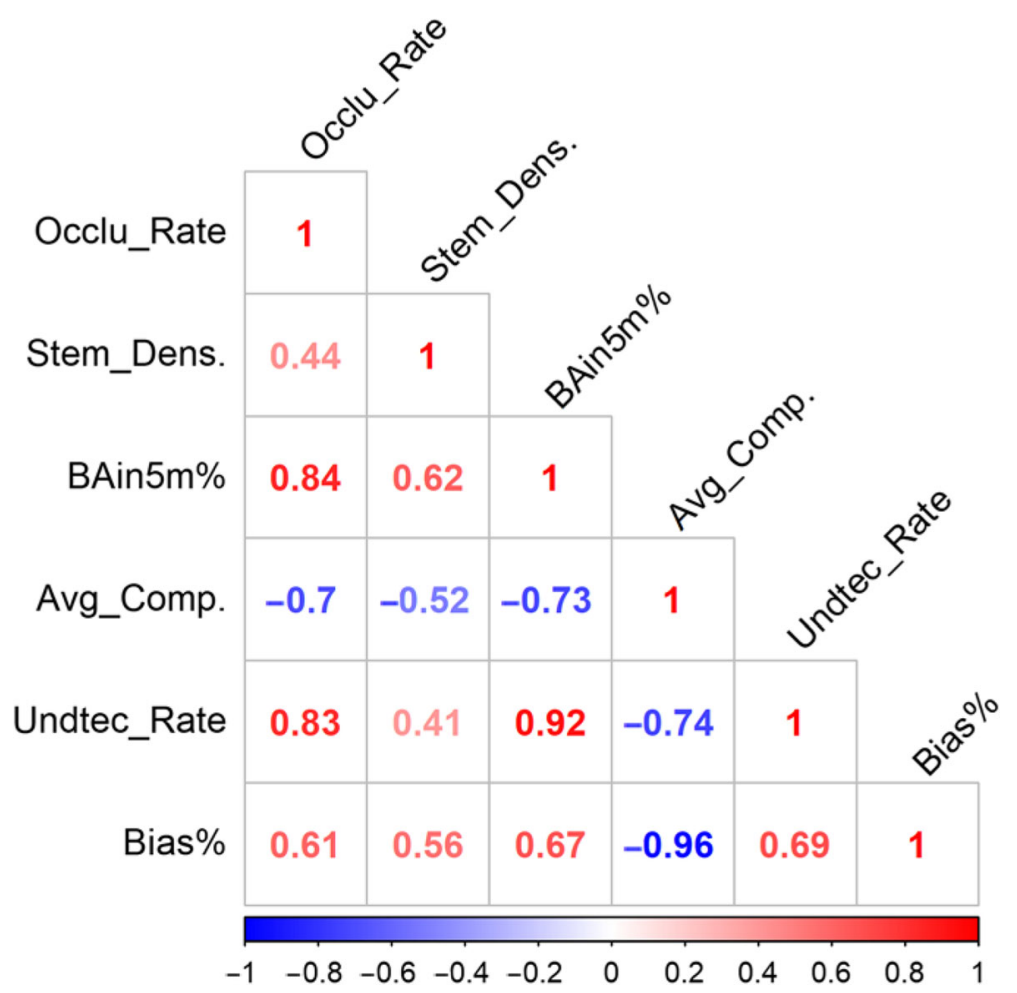

Fig. 8 The correlation matrix of all occlusion indicators and accuracy indices. The Occlu_Rate, Stem_Dens., Avg_Comp. and Undtec_Rate denote the occlusion rate, stem density, average completeness and undetected rate, respectively

rate, mean relative bias and mean completeness were measured in different distance sections. The distance sections were set with a 5-m interval in this study. Figure 9 shows the correlation between the scanning distance and accuracy. It shows that the undetected rate is increased with an increased scanning distance, and the correlation coefficient is 0.99 . The undetected rate within $10 \mathrm{~m}$ is under $10 \%$, and then rapidly increased to $25 \%$ within 10 to $15 \mathrm{~m}$. The completeness and relative bias are also highly related to the scanning distance; the correlation coefficient is -0.85 and 0.85 , respectively. The results indicate that the scanning distance is a significant factor in the stem curve retrieving.

\section{Influence of the different occlusion sources}

Based on the stem maps, the occlusion from S1 can be directly recognized. We counted the number of undetected stems and partly occluded stems from S1. In
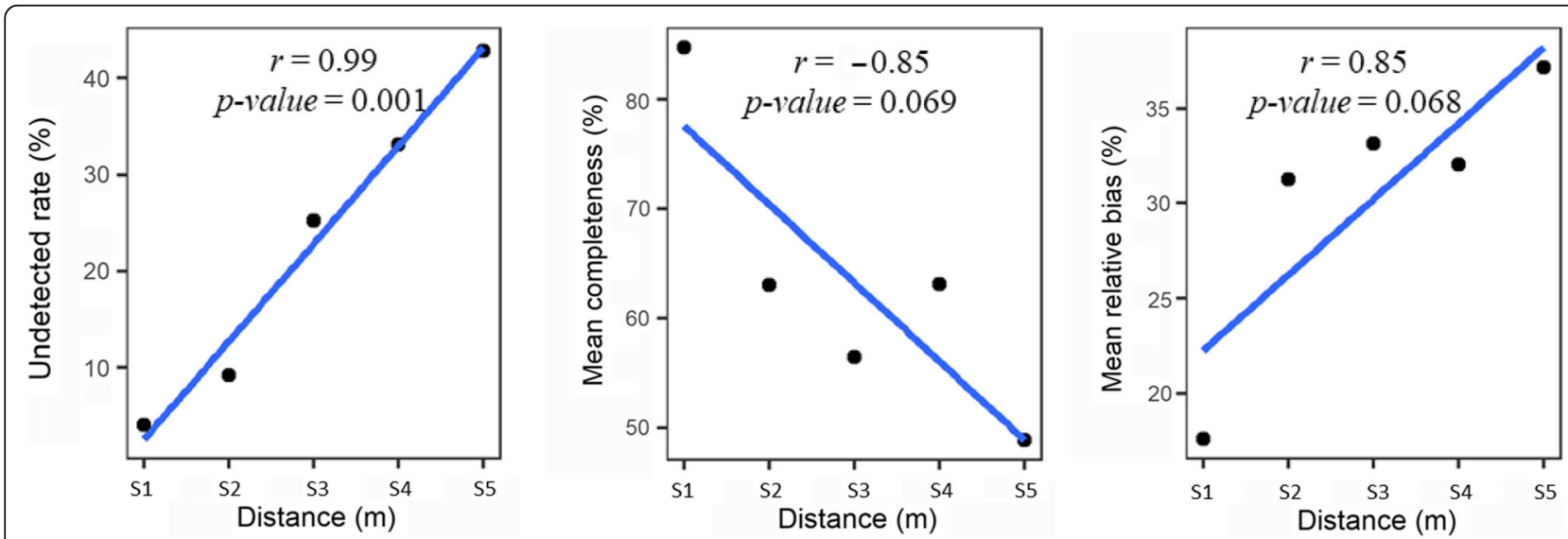

Fig. 9 The influence of scanning distance on the accuracy indexes. S1 to S5 denote the distance sections, (0-5), (5-10), (10-15), (15-20), (20-25) m, respectively 
general, two-thirds of the undetected stems are caused by $\mathrm{S} 1$, as shown in Table 3. Meanwhile, some stems are partly occluded, which led to the defective point cloud data of stems and unreal stem diameters. It shows that partial occlusions seriously affected the accuracy of measured stem diameters. The underestimation of stem diameters exceeded $10 \mathrm{~cm}$ in most plots, and the mean value is approximately $13.6 \mathrm{~cm}$.

Table 4 shows that approximately one-third of all undetected stems was caused by S2. In addition, the S2 are the main factors for the seriously occluded stems which have very low completeness $(<5 \%)$ of the stem curves.

\section{Discussion}

\section{The capacity of retrieving stem curve from single-scan} TLS data

The capacity of a single-scan TLS data to retrieve stem curves is mainly dependent on the level of data deficient caused by occlusion. The results showed that the occlusions significantly affect stem curve estimates. On average, approximately $16.4 \%$ of stems were undetected in each plot, the mean completeness of the stem curve was $65.29 \%$, and the underestimation of the diameters was approximately $4 \mathrm{~cm}$ when compared with the multi-scan datasets. Among the test plots, only one plot obtained a mean bias fewer than $2 \mathrm{~cm}$ and the relative bias was less than $10 \%$. The largest mean bias and relative bias reached $8.464 \mathrm{~cm}$ and $46.93 \%$, respectively. A plot with a larger occlusion rate is more likely has lower accuracy on retrieving stem curves. The undetected rate was similar to the conclusion of the international TLS benchmarking project (Liang et al. 2018), in which the stem-detection rate was improved by

Table 3 The occlusion effect caused by tree stems

\begin{tabular}{|c|c|c|c|c|}
\hline \multirow{2}{*}{$\begin{array}{l}\text { Plot } \\
\text { ID }\end{array}$} & \multicolumn{2}{|c|}{ Totally blocked stems } & \multicolumn{2}{|c|}{ Partly blocked stems } \\
\hline & Number & Percentage $^{a}$ & Number & Mean bias $(\mathrm{cm})$ \\
\hline 1 & 1 & $1 / 3$ & 1 & -7.4 \\
\hline 4 & 4 & $2 / 3$ & 5 & -8.6 \\
\hline 5 & 7 & $1 / 4$ & 1 & -8.3 \\
\hline 6 & 10 & $2 / 3$ & 5 & -19.2 \\
\hline 10 & 9 & $2 / 3$ & 3 & -18.2 \\
\hline 12 & 11 & 55 & 8 & -10.5 \\
\hline 16 & 4 & 1 & 6 & -12.8 \\
\hline 18 & 2 & 1 & 4 & -24.0 \\
\hline 19 & 2 & $2 / 3$ & 0 & - \\
\hline 20 & 10 & $1 / 4$ & 7 & -7.8 \\
\hline 22 & 3 & 1 & 1 & -19.3 \\
\hline Mean & - & $2 / 3$ & - & -13.6 \\
\hline
\end{tabular}

Note: ${ }^{\text {T}}$ The percentage of the undetected stems caused by tree stems in all undetected stems
Table 4 The occlusion effect caused by non-stem components

\begin{tabular}{|c|c|c|c|}
\hline \multirow{2}{*}{$\begin{array}{l}\text { Plot } \\
\text { ID }\end{array}$} & \multicolumn{2}{|c|}{ Totally blocked stems } & \multirow{2}{*}{$\begin{array}{l}\text { Seriously incomplete stems } \\
\text { Number }\end{array}$} \\
\hline & Number & Percentage $^{a}$ & \\
\hline 1 & 2 & $2 / 3$ & 4 \\
\hline 4 & 2 & $1 / 3$ & 1 \\
\hline 5 & 19 & $3 / 4$ & 7 \\
\hline 6 & 5 & $1 / 3$ & 13 \\
\hline 10 & 4 & $1 / 3$ & 10 \\
\hline 12 & 9 & $4 / 9$ & 12 \\
\hline 16 & 0 & 0 & 0 \\
\hline 18 & 0 & 0 & 1 \\
\hline 19 & 1 & $1 / 3$ & 0 \\
\hline 20 & 29 & $3 / 4$ & 8 \\
\hline 22 & 0 & 0 & 2 \\
\hline Mean & - & $1 / 3$ & - \\
\hline
\end{tabular}

Note: ${ }^{a}$ The percentage of the undetected stems caused by non-stem components in all undetected stems. The seriously incomplete stems are the stems where the completeness of the retrieved stem curves is less than $5 \%$

approximately $20 \%$ when using the multi-scan approach. However, they reported no significant improvements in the parameter estimations. The discrepancy indicates that, to some extent, a good method of stem modeling can overcome data deficient. It should be noted that the occlusion effect also exists in the multi-scan datasets, which makes the detection rate of stems and the completeness of the retrieved stem curve not completely accurate. In multi-scan datasets, according to the results from international TLS benchmarking project, the best detection rate of stems was approximately $90 \%$ in the easy plots and approximately $66 \%$ in the difficult plots. In addition, the best completeness rate reached $97 \%$ and $88 \%$ for the easy and difficult plots, respectively. According to the results in this study and the conclusions made in previous research, the singlescan TLS data can be used for the stem curve retrieving in small plots for rapid forest inventory tasks. In this study, the stems within $10 \mathrm{~m}$ from the scanning center were measured accurately (Fig. 9). Since the stem density of the plots is diverse in our experiments, we suggested that $10 \mathrm{~m}$ is an appropriate extent size of plot for most forest condition for using single-scan mode. This result may enlighten the future studies that combining traditional forest statistical methods and single-scan TLS data of small plots to survey forest resources. Besides downsizing the plot size, we can improve the accuracy of retrieving stem curves by selecting the plots with lower occlusion rates $(<35 \%)$ or optimizing the scanning position for a lower PBA. The occlusion rates and PBA can be predicted using airborne remote sensing techniques 
before TLS data collection. Therefore, further studies may focus on the optimization of the scanner position of TLS.

\section{Effectiveness of the occlusion rate to predict the estimation accuracy}

As a general descriptor, the occlusion rate was highly related to the plot-level accuracy assessment index, such as undetected rate, for which the correlation coefficient reached 0.828 . The correlation was followed by -0.696 and 0.607 for average completeness and relative bias, respectively. This is consistent with the report of the international TLS benchmarking project where the stand condition (i.e., easy, medium, and difficult) mainly increases the difficulty of stem detection. The decline in correlation was considered reasonable because the influence factors became more complicated and random for the completeness and the bias. The occlusion rate is easy-to-calculate and highly related to the estimation accuracy, which can be used for dataset selection before calculating the stem curve. It is worth noting that the occlusion rate is a macro indicator derived from the $2 \mathrm{D}$ image. Therefore, it is not always consistent with the real level of occlusions. In some plots with small trees and very dense leaves, the occlusion rate is low but the level of occlusions is high, because of the presence of the dense leaves in 3D space. A 3D occlusion index may describe the occlusion level more precisely and has a higher correlation to the stem curve retrieving. However, it could be more complex which we cannot obtain easily. In future studies, the 3D occlusion index and its relations to the optimized scanning positions could be explored by simulating the TLS data of a virtual forest (Hämmerle et al. 2017). The advantage of our proposed occlusion rate is easy-to-obtain and easy-to-calculate. The occlusion rate is a device-independent index that only determined by the stand condition and scanning position, which means that using different devices with different setting up parameters will obtain similar occlusion rates for the same forest plot.

\section{Factors influencing the estimation accuracy of the stem curve}

The percentage of basal area within $\mathrm{N}$ meters from the scanning center (PBA) was found to be more strongly correlated with the accuracy indices compared to the stem density. The correlation between the PBA and the undetected rate reached 0.92 , which was much higher than that for stem density $(r=0.41)$. In regard to the depiction of the occlusion effect, PBA is more effective because more stems are close to the scanning center and stems with larger diameters will have a higher probability to occlude other stems during data collection. In contrast, the stem density only reflects the overall distribution of stems without the information about stem diameters. However, they are not independent occlusion factors. Stem density influences PBA to some extent as evidenced by our tests (Fig. 8); the correlation coefficient between them was 0.62 .

Previous studies suggest that the scanning distance mainly affects stem detection and has less impact on the estimation accuracy of basic forest inventory parameters, such as the diameter at breast height (DBH) (Pueschel 2013). Some research reported a steadily decreasing rate in tree mapping as the scanning distance increases and the tree mapping accuracy decreased from $85 \%$ to $73 \%$ if the plot radius increases from 5 to $10 \mathrm{~m}$ (Liang et al. 2012). In this study, we found that the scanning distance is a significant factor that influences the estimation accuracy of stem curves, not only the undetected rate but also the completeness and bias. It can be explained that the retrieving of stem curves is more easily affected by the occlusions than other forest inventory parameters because the upper stems are more challenging to measure.

The key to recognizing the source of occlusion is a stem map with position and DBH of tree stems. Based on the stem map and the propagation of laser beams, we can simulate the inter-visibility of a plot between stems. In this study, the stem maps were derived from the multi-scan TLS datasets. However, the inter-visibility analysis should be performed before the TLS data collection. In practice, the stem map can be obtained by using other remote sensing approaches, such as unmanned aerial vehicles (UAV) and TLS data simulation. The analysis of stem map and the arrangement of scanning positions based on the UAV imagery and TLS data simulation, which is a worthy direction for further studies.

\section{Conclusions}

The single-scan terrestrial laser scanning is a time-efficient approach for the estimation of stem curves. However, occlusion effect severely affects the accuracy of retrieved stem curves. In this study, we proposed an effective and easy-tocalculate index, the occlusion rate, to quantify the occlusion degree of single-scan TLS data. The occlusion rate of a plot is highly related to the accuracy of retrieved stem curves and the distribution pattern of stem density. To describe the distribution pattern of basal area, we introduced the PBA which is more effective in determining the occlusions than stem density. In addition, we found that the accuracy of the estimated stem curve was decreased with increasing scanning distance with high correlations $(r=0.85-0.99)$. Based on the conclusions drawn from the findings of this study, we suggested that to use single-scan TLS data to accurately estimate the stem curve in a small forest plot $(<10 \mathrm{~m})$ or the plot with lower occlusion rate, such as less than $35 \%$ in test datasets. The findings from this study are useful for guiding the practice of retrieving forest parameters using single-scan TLS data. 


\section{Abbreviations}

CC: Connected component; CSF: Cloth simulation filtering; DBH: Diameter at breast height; FGl: Finish geospatial research institute; PBA: The percentage of basal area within n meters from the scanning center; RHT: Randomized hough transform; RMSE: Root mean square error; TLS: Terrestrial laser scanning

\section{Acknowledgments}

Not applicable.

\section{Authors' contributions}

PW designed the experiments, processed the data and analyzed the results. PW, TW and WZ coordinated the manuscript preparation. All authors contributed to the manuscript writing and editing. All authors read and approved the final manuscript.

\section{Funding}

This work was supported by the National Natural Science Foundation of China (Grant Nos. 41671414, 41971380, 41331171 and 41171265) and the National Key Research and Development Program of China (No. 2016YFB0501404).

\section{Availability of data and materials}

The datasets used and/or analyzed during the current study are available from the corresponding author on reasonable request.

\section{Ethics approval and consent to participate}

The subject has no ethic risk.

\section{Consent for publication}

Not applicable.

\section{Competing interests}

The authors declare that they have no competing interests.

\section{Author details}

${ }^{1}$ State Key Laboratory of Remote Sensing Science, Jointly Sponsored by Beijing Normal University and Institute of Remote Sensing and Digital Earth of Chinese Academy of Sciences; Beijing Engineering Research Centre for Global Land Remote Sensing Products, Institute of Remote Sensing Science and Engineering, Faculty of Geographical Science, Beijing Normal University, Beijing 100875, China. ${ }^{2}$ Changjiang River Scientific Research Institute (CRSRI), Wuhan 430010, China. ${ }^{3}$ Faculty of Geo-Information Science and Earth Observation (ITC), University of Twente, AE 7500 Enschede, The Netherlands. ${ }^{4}$ Department of Remote Sensing and Photogrammetry, Finnish Geospatial Research Institute, 02431 Masala, Finland. ${ }^{5}$ Department of Environmental Science, Macquarie University, Macquarie Park 2106, Australia.

Received: 13 June 2019 Accepted: 24 September 2019

Published online: 14 October 2019

\section{References}

Abegg M, Kükenbrink D, Zell J, Schaepman ME, Morsdorf F (2017) Terrestrial laser scanning for forest inventories-tree diameter distribution and scanner location impact on occlusion. Forests 8(6):184

Astrup R, Ducey MJ, Granhus A, Ritter T, von Lupke N (2014) Approaches for estimating stand-level volume using terrestrial laser scanning in a single-scan mode. Can J For Res 44(6):666-676

Burkhart HE, Tomé M (2012) Modeling forest trees and stands. Springer Science \& Business Media, The Netherlands

Clark NA, Wynne RH, Schmoldt DL (2000) A review of past research on dendrometers. For Sci 46:570-576

Corral-Rivas JJ, Diéguez-Aranda U, Rivas SC, Dorado FC (2007) A merchantable volume system for major pine species in El Salto, Durango (Mexico). For Ecol Manag 238:118-129

Dassot M, Constant T, Fournier M (2011) The use of terrestrial LiDAR technology in forest science: application fields, benefits and challenges. Ann For Sci 68: 959-974

Drew DM, Downes GM (2018) Growth at the microscale: long term thinning effects on patterns and timing of intra-annual stem increment in radiata pine. Forest Ecosyst 5:32. https://doi.org/10.1186/s40663-018-0153-z
Girardeau-Montaut D (2018) CloudCompare-3D point cloud and mesh processing software (version 2.10.Beta). GPL software http://www. cloudcompare.org/. Accessed on 16 Oct 2018

Hämmerle M, Lukač N, Chen K-C, Zs K, Wang C-K, Anders K, Höfle B (2017) Simulating various terrestrial and UAV Lidar scanning configurations for understory forest structure modelling. ISPRS Ann Photogramm Remote Sens Spat Inf Sci IV-2(W4):59-65. https://doi.org/10.5194/isprs-annals-IV-2W4-59-2017

Kalantari H, Fallah A, Hodjati SM, Parsakhoo A (2012) Determination of the most appropriate form factor equation for Cupresus sempervirence $L$. var horizentalis in the north of Iran. Adv Appl Sci Res 3:644-648

Kankare V, Holopainen M, Vastaranta M, Puttonen E, Yu X, Hyyppä J, Vaaja M, Hyyppä H, Alho P (2013) Individual tree biomass estimation using terrestrial laser scanning. ISPRS J Photogramm Remote Sens 75:64-75. https://doi.org/ 10.1016/j.isprsjprs.2012.10.003

Kelbe D, van Aardt J, Romanczyk P, van Leeuwen M, Cawse-Nicholson K (2015) Single-scan stem reconstruction using low-resolution terrestrial laser scanner data. IEEE J Sel Top Appl Earth Obs Remote Sens 8:3414-3427. https://doi. org/10.1109/JSTARS.2015.2416001

Liang X, Hyyppä J, Kaartinen H, Lehtomaki M, Pyorala J, Pfeifer N, Holopainen M, Brolly G, Pirotti F, Hackenberg J, Huang HB, Jo HW, Katoh M, Liu LX, Mokros M, Morel J, Olofsson K, Poveda-Lopez J, Trochta J, Wang D, Wang JH, Xi ZX, Yang BS, Zheng G, Kankare V, Luoma V, Yu XW, Chen L, Vastaranta M, Saarinen N, Wang YS (2018) International benchmarking of terrestrial laser scanning approaches for forest inventories. ISPRS J Photogramm Remote Sens 144:137-179. https://doi.org/10.1016/j.isprsjprs.2018.06.021

Liang X, Hyyppä J, Kankare V, Holopainen M (2013) Stem curve measurement using terrestrial laser scanning. IEEE Trans Geosci Remote Sens 52:1739-1748

Liang X, Litkey P, Hyyppa J, Kukko A, Kaartinen H, Holopainen M (2008) Plot-level trunk detection and reconstruction using one-scan-mode terrestrial laser scanning data. In: 2008 international workshop on earth observation and remote sensing applications, Beijing, China. IEEE, pp 1-5

Liang X, Litkey P, Hyyppa J et al (2012) Automatic stem mapping using singlescan terrestrial laser scanning. IEEE Trans Geosci Remote Sens 50:661-670. https://doi.org/10.1109/TGRS.2011.2161613

Liang XL, Wang YS, Pyorala J, Lehtomaki M, Yu XW, Kaartinen H, Kukko A, Honkavaara E, Issaoui AEl, Nevalainen O, Vaaja M, Virtanen JP, Katoh M, Deng SQ (2019) Forest in situ observations using unmanned aerial vehicle as an alternative of terrestrial measurements. Forest Ecosyst 6:20. https:/doi.org/10.1186/s40663-019-0173-3

Lovell JL, Jupp DLB, Newnham GJ, Culvenor DS (2011) Measuring tree stem diameters using intensity profiles from ground-based scanning lidar from a fixed viewpoint. ISPRS J Photogramm Remote Sens 66:46-55

Lundgren C (2000) Predicting log type and knot size category using external log shape data from a 3D log scanner. Scand J For Res 15:119-126

Olofsson K, Olsson H (2017) Estimating tree stem density and diameter distribution in single-scan terrestrial laser measurements of field plots: a simulation study. Scand J For Res:1-13. https:/doi.org/10.1080/02827581.2017.1368698

Özçelik R, Wiant HV Jr, Brooks JR (2008) Accuracy using xylometry of log volume estimates for two tree species in Turkey. Scand J For Res 23:272-277

Pauly M, Gross M, Kobbelt LP (2002) Efficient simplification of point-sampled surfaces. In: Proceedings of the conference on Visualization'02. IEEE computer society, pp 163-170

Pueschel P (2013) The influence of scanner parameters on the extraction of tree metrics from FARO photon 120 terrestrial laser scans. ISPRS J Photogramm Remote Sens 78:58-68

Pueschel P, Newnham G, Rock G et al (2013) The influence of scan mode and circle fitting on tree stem detection, stem diameter and volume extraction from terrestrial laser scans. ISPRS J Photogramm Remote Sens 77:44-56. https://doi.org/10.1016/j.isprsjprs.2012.12.001

Stovall AEL, Vorster AG, Anderson RS et al (2017) Non-destructive aboveground biomass estimation of coniferous trees using terrestrial LiDAR. Remote Sens Environ 200:31-42

Trochta J, Král K, Janík D, Adam D (2013) Arrangement of terrestrial laser scanner positions for area-wide stem mapping of natural forests. Can J For Res 43: 355-363. https://doi.org/10.1139/cjfr-2012-0347

Trochta J, Krůček M, Vrška T, Král K (2017) 3D Forest: an application for descriptions of three-dimensional forest structures using terrestrial LiDAR. PLoS One 12:e0176871

Van der Zande D, Hoet W, Jonckheere I et al (2006) Influence of measurement set-up of ground-based LiDAR for derivation of tree structure. Agric For Meteorol 141:147-160 
Watt PJ, Donoghue DNM (2005) Measuring forest structure with terrestrial laser scanning. Int J Remote Sens 26:1437-1446

West P (2009) Trees and forest measurement. Springer, Berlin, Heidelberg Wilkes P, Lau A, Disney M et al (2017) Data acquisition considerations for terrestrial laser scanning of forest plots. Remote Sens Environ 196:140-153

Xu L, Oja E (1993) Randomized Hough transform (RHT): basic mechanisms, algorithms, and computational complexities. CVGIP Image Underst 57:131-154

Xu L, Oja E, Kultanen P (1990) A new curve detection method: randomized Hough transform (RHT). Pattern Recogn Lett 11:331-338

Yu X, Liang X, Hyyppä J et al (2013) Stem biomass estimation based on stem reconstruction from terrestrial laser scanning point clouds. Remote Sens Lett 4:344-353

Zhang W, Chen Y, Wang H et al (2016a) Efficient registration of terrestrial LiDAR scans using a coarse-to-fine strategy for forestry applications. Agric For Meteorol 225:8-23

Zhang W, Qi J, Wan P et al (2016b) An easy-to-use airborne LiDAR data filtering method based on cloth simulation. Remote Sens 8:501. https://doi.org/10.3390/rs8060501

Zhang W, Wan P, Wang T et al (2019) A novel approach for the detection of standing tree stems from plot-level terrestrial laser scanning data. Remote Sens 11(2):211. https://doi.org/10.3390/rs11020211

\section{Submit your manuscript to a SpringerOpen ${ }^{\circ}$ journal and benefit from:}

- Convenient online submission

- Rigorous peer review

- Open access: articles freely available online

- High visibility within the field

- Retaining the copyright to your article

Submit your next manuscript at $\boldsymbol{\triangleright}$ springeropen.com 